\title{
Determinación y análisis estadístico de la composición de los gases producidos en un piloto de recobro mejorado
}

\section{Determination and statistical analysis of the composition of the gases produced in an Enhanced Oil Recovery Pilot}

\section{Determinação e análise estatística da composição de gases produzidos num piloto de recuperação avançada de petróleo}

\author{
José Manuel Díaz-Molina1*; Andrea Carolina Morales-Toscano; Marisol Fernández-Rojas; \\ Nelson Briceño-Gamba'; Deicy Villalba-Rey; María del Rosario-Sánchez² \\ ${ }^{1}$ Facultad de Ciencias Básicas e Ingeniería. Universidad de los Llanos (Unillanos), km 12 vía Puerto López, \\ Villavicencio, Colombia \\ ${ }^{2}$ Instituto Colombiano de Petróleo. Ecopetrol S. A., km 7 vía Piedecuesta, Santander, Colombia \\ *nbriceno@unillanos.edu.co
}

Fecha recepción: 11 de abril de 2019 Fecha aceptación: 27 de junio de 2019

\begin{abstract}
Resumen
La industria petrolera en Colombia tiene el desafío de la explotación de yacimientos con crudo pesado y extrapesado. Por este motivo, en el país se han implementado métodos de recobro mejorado (EOR) que suelen tener altos índices de producción. La inyección de aire es uno de los métodos de recobro mejorado que permite alcanzar altos índices de producción. La planta Piloto de Inyección de Aire para Recobro (PIAR), ubicada en Chichimene (Meta), es pionera en el mundo por implementar este método en una reserva de crudo extrapesado $(\mathrm{API}<10)$ de alta profundidad. El monitoreo de las propiedades fisicoquímicas de los fluidos generados durante este proceso contribuye a asegurar el correcto funcionamiento operativo del piloto. En este trabajo se determinó por cromatografía de gases (GC) la composición de los gases de producción de 16 pozos ubicados en el área del piloto. Se encontró principalmente metano $(68,5 \pm 7,1$ $\% \mathrm{~mol}), \mathrm{CO}_{2}(11,4 \pm 5,6 \% \mathrm{~mol})$ y $\mathrm{N}_{2}(6,7 \pm 4,2 \% \mathrm{~mol})$. La composición de los gases fue disímil en toda el área, por lo que se hicieron análisis multivariados, análisis de componentes principales (ACP)

y análisis de conglomerados (AC), para evaluar las similitudes entre los gases de los pozos. Como resultado, los pozos fueron organizados en tres grupos por similitud: un grupo con los niveles más altos de metano $(75,8 \% \mathrm{~mol})$; el segundo grupo con $\mathrm{N}_{2}(11,9 \% \mathrm{~mol})$ y un tercer grupo con los valores más altos de $\mathrm{CO}_{2}(20,1 \% \mathrm{~mol})$, conformado por tres pozos. Estos grupos fueron graficados en un mapa y se observó que se encuentran en áreas muy definidas dentro del piloto.
\end{abstract}

Palabras clave: Inyección de Aire; Cromatografía de Gases; Crudo Extrapesado; Recobro Mejorado.

\section{Abstract}

The oil industry in Colombia has the challenge of exploiting deposits with heavy and extra-heavy oil. For this reason, Enhanced Oil Recovery methods (EOR) have been implemented in the country, looking for high production rates. Air injection is one of the EOR methods that allows to achieve high production rates. The Air Injection Pilot (PIAR) at Chichimene (Meta) is a pioneer in the world for implementing this method in a reserve of extra heavy oil $(\mathrm{API}<10)$ in a high depth. The monitoring of the physicochemical properties of the fluids generated during this process helps to ensure the correct operational functioning of the pilot. In this work, the gas composition from 16 wells located in the pilot area was determined by gas chromatography (GC). Methane was found mainly $(68.5 \pm 7.1 \% \mathrm{~mol})$, followed by $\mathrm{CO}_{2}(11.4 \pm 5.6 \% \mathrm{~mol})$

Cita: Díaz Molina JM, Morales Toscano AC, Fernández Rojas M, Briceño-Gamba N, Villalba Rey D, Sánchez MR. Determination and statistical analysis of the composition of the gases produced in an Enhanced Oil Recovery Pilot. rev.ion. 2019;32(1):63-73. doi:10.18273/revion.v32n1-2019006 
and $\mathrm{N}_{2}(6.7 \pm 4.2 \% \mathrm{~mol})$. The composition of the gases was dissimilar throughout the area, so Principal Component Analysis (PCA) and Cluster Analysis (CA) were made to assess the similarities between the gases of the wells. As a result, the wells were organized into three groups by similarity: a group with the highest methane levels $(75.8 \% \mathrm{~mol})$; the second group with $\mathrm{N}_{2}(11.9 \% \mathrm{~mol})$; and a third group with the highest $\mathrm{CO}_{2}$ values $(20.1 \% \mathrm{~mol})$, consisting of three wells. These groups were plotted on a map and it was noted that they are in defined areas within the pilot.

Keywords: Air Injection; Gas Chromatography; Extra Heavy Oil; Enhanced Oil Recovery.

\section{Resumo}

A indústria petroleira em Colômbia tem o desafio da exploração de depósitos com petróleo pesado e extrapesado. Por este motivo, no país tem sido implementados métodos de recuperação avançada de petróleo (EOR) os quais apresentam altos índices de produção. A injeção de ar é um dos métodos de recuperação que permite alcançar altos índices de produção. A planta piloto de injeção de ar para recobro (PIAR) no campo Chichimene (Meta) é pioneira no mundo por implementar este método numa reserva de petróleo extrapesado $(\mathrm{API}<10)$ de alta profundidade. O monitoramento das propriedades físico-químicas dos fluidos gerados durante este processo contribui no asseguramento do correto funcionamento operativo do piloto. Neste trabalho determinou-se por cromatografia de gases (GC) a composição dos gases de produção de 16 poços localizados na área do piloto. Encontrou-se principalmente metano $(68,5 \pm 7,1 \% \mathrm{~mol}), \mathrm{CO}_{2}(11,4 \pm 5,6 \% \mathrm{~mol})$ e $\mathrm{N}_{2}(6,7 \pm 4,2 \% \mathrm{~mol})$. A composição dos gases foi variável em toda a área, por tanto foram feitos análise multivariados análise de componentes principais (ACP) e análise de conglomerados (AC) para avaliar as semelhanças entre os gases dos poços. Como resultado os poços se organizaram em 3 grupos por similaridade: um grupo com os níveis mais altos de metano ( $75,8 \% \mathrm{~mol})$; o segundo grupo com $\mathrm{N}_{2}(11,9 \% \mathrm{~mol})$; e um terceiro grupo com os valores mais altos de $\mathrm{CO}_{2}(20,1 \% \mathrm{~mol})$, conformado por três poços. Estes grupos foram plotados em um mapa e observou-se que se encontram em áreas bem definidas dentro do piloto.

Palavras-chave: Injeção de Ar; Cromatografia de Gases (GC); Petróleo Extrapesado; Recuperação Avançada de Petróleo.

\section{Introducción}

Las reservas de crudo medios a livianos son cada día más escasas y la demanda energética sigue subiendo a nivel mundial. Esta situación ha hecho que la industria petrolera se interese por la explotación de crudos pesados, los cuales representan un $70 \%$ de las reservas del mundo [1]. En Colombia, se estima que de las reservas de hidrocarburos halladas en el país, un $60 \%$ corresponde a crudos pesados [2]. Dentro de los métodos convencionales de extracción de hidrocarburos se encuentran los métodos de recobro primario (RP) y recobro secundario (RS); estos son implementados en las fases tempranas de la explotación de los yacimientos [3]. Los métodos RP son los primeros en desarrollarse; estos extraen el crudo usando la energía natural de la reserva y logran alcanzar porcentajes estimados de recobro de hasta un $10 \%$. Posterior a esto, los métodos RS son ejecutados. La inyección de vapor de agua o la inyección de agua caliente suelen ser usadas en esta etapa y se han reportado casos en los cuales con estos métodos se alcanzan valores de recobro cercanos al 50 \%. Aun así, los métodos de RP y RS suelen ser ineficientes en yacimientos con crudos pesados, los cuales tienen una gravedad API inferior a $22^{\circ}$ y altas viscosidades $(>1000 \mathrm{cP})$, por lo cual se dificulta alcanzar estimados de recobro que justifiquen su inversión [3]. Por lo tanto, la explotación de este recurso se hace a partir de métodos de recobro mejorado (Enhance Oil Recovery, EOR), los cuales necesitan de una alta inversión económica y tecnológica [3]. Los métodos EOR fueron adoptados con el fin de recuperar el crudo dentro de reservas parcialmente explotadas [4]. Estas técnicas se enfocan en cambiar las propiedades de los hidrocarburos, como densidad o viscosidad, o incrementar la presión diferencial en los pozos por medio de la inyección de otros fluidos al yacimiento [4]. Una vez se decide implementar un método de recobro mejorado en un yacimiento, es necesario conocer ciertos aspectos del crudo para así seleccionar el método más apropiado de recobro. La selección del método EOR se hace teniendo 
en cuenta la viscosidad del crudo y la profundidad de la reserva, principalmente [3]. Actualmente, en Colombia, es necesario implementar métodos EOR para mantener la competitividad en el mercado y afrontar desde ahora la futura escasez de crudos medios o livianos. En este sentido, se han implementado diferentes proyectos de desarrollo tecnológico dentro de los cuales se destacan el Piloto de Inyección de Agua Mejorada y el Piloto de Inyección de Aire para Recobro (PIAR), ambos ubicados en la cuenca de los llanos orientales, una de las zonas más productivas del país [2]. EI PIAR se desarrolla en el campo Chichimene (Meta, Colombia); este campo se caracteriza por producir crudo extrapesado, el cual representa el $11.5 \%$ de la producción total de crudo diaria en Colombia [5]. El factor de recobro para este crudo, con viscosidad superior a $2000 \mathrm{cP}$ y gravedad API entre $7^{\circ}$ y $10^{\circ}$, puede estar cerca del $10 \%$ [6]; por medio de la inyección de aire, se espera que este porcentaje incremente alrededor de $50 \%$ considerando los resultados obtenidos en proyectos exitosos de este tipo $[7,8]$. En Colombia, un piloto similar a este se desarrolló en el campo Quifa, en los llanos orientales, en el cual se logró un incremento en el factor recobro del crudo [10]. Sin embargo, el PIAR es pionero en el mundo ya que la formación de San Fernando se encuentra a una profundidad superior a 2000 metros, lo que la convierte en la reserva de crudo extrapesado más profunda del mundo [11].

La inyección de aire consiste en introducir aire a través de un pozo inyector e inducir una combustión in situ (CIS), lo cual genera un frente de combustión alimentado por las interacciones del crudo y el oxígeno del aire inyectado. Este proceso libera calor, eleva la temperatura del yacimiento y disminuye la viscosidad del crudo, por lo cual se facilita el flujo del crudo en los pozos productores [3]. Como consecuencia de la ignición, muchas reacciones suelen llevarse a cabo dentro del yacimiento, por lo que las propiedades físicas y químicas del crudo pueden variar significativamente. Esto hace relevante el continuo monitoreo de variables como la composición química de los fluidos generados en la producción (agua, gas y crudo), para obtener información sobre los procesos fisicoquímicos que suceden en el interior del yacimiento y poder tomar decisiones sobre algunos parámetros operativos del piloto [7,8]. Dentro de los análisis para monitorear la CIS se tiene la cromatografía de gases (GC). Esta es una de las técnicas más usadas ya que permite determinar la composición del gas de producción, fluido que más rápido lleva información a la superficie, por lo cual se facilita la atención de las alertas encontradas. Este análisis permite localizar el frente de combustión, confirmar la ignición en el yacimiento, determinar si la combustión es completa e incompleta, tomar decisiones acerca de la tasa de inyección de aire y generar alertas sobre posibles problemas de seguridad ambiental e industrial [8]. Por lo tanto, antes de iniciar la inyección de aire, es importante establecer la influencia del pozo inyector en los demás pozos del yacimiento y establecer una línea base de la composición de los gases producidos en estos [6]. En este sentido, en el año 2015 se realizó una prueba de conectividad con nitrógeno (PCN) a partir de la cual se determinaron los pozos de influencia del pozo inyector [6]. En este trabajo se determinó por GC la composición de los gases de producción de los pozos influenciados posterior a la prueba PCN del año 2015. Así mismo, se realizaron análisis estadísticos multivariados de los datos usando boxplot, diagramas de componentes principales y conglomerados; con base en estos resultados, se elaboró la línea base de la composición de los gases.

\section{Materiales y métodos}

\section{Área de estudio}

El Piloto de Inyección de Aire para Recobro (PIAR) se encuentra dentro del campo petrolero Chichimene, a $12 \mathrm{~km}$ al sureste del municipio de Acacías, en el departamento del Meta. Este piloto abarca pozos de las unidades productivas T2 (arenas del periodo Terciario) y K1 (arenas del periodo Cretácico) de la reserva de San Fernando, la cual cuenta con una extensión superficial de 750 $\mathrm{hm}^{2}$ y un área de yacimiento de $374 \mathrm{hm}^{2}$.

\section{Muestreo}

Durante un mes se muestrearon una vez por semana 16 de los 26 pozos del área de influencia del piloto: dos de primera línea (P1 y P2), dos de segunda línea ( $P 3$ y $P 4)$, tres de tercera línea ( $P 5$, P6 y P7) y nueve pozos externos (P8 al P16). El mapa del arreglo de pozos es mostrado en la figura 1. El muestreo se realizó en los anulares de las cabezas de los pozos usando cilindros Swagelok de 500 cc en acero DOT3A1800, pasivados con Sulfinert@. 


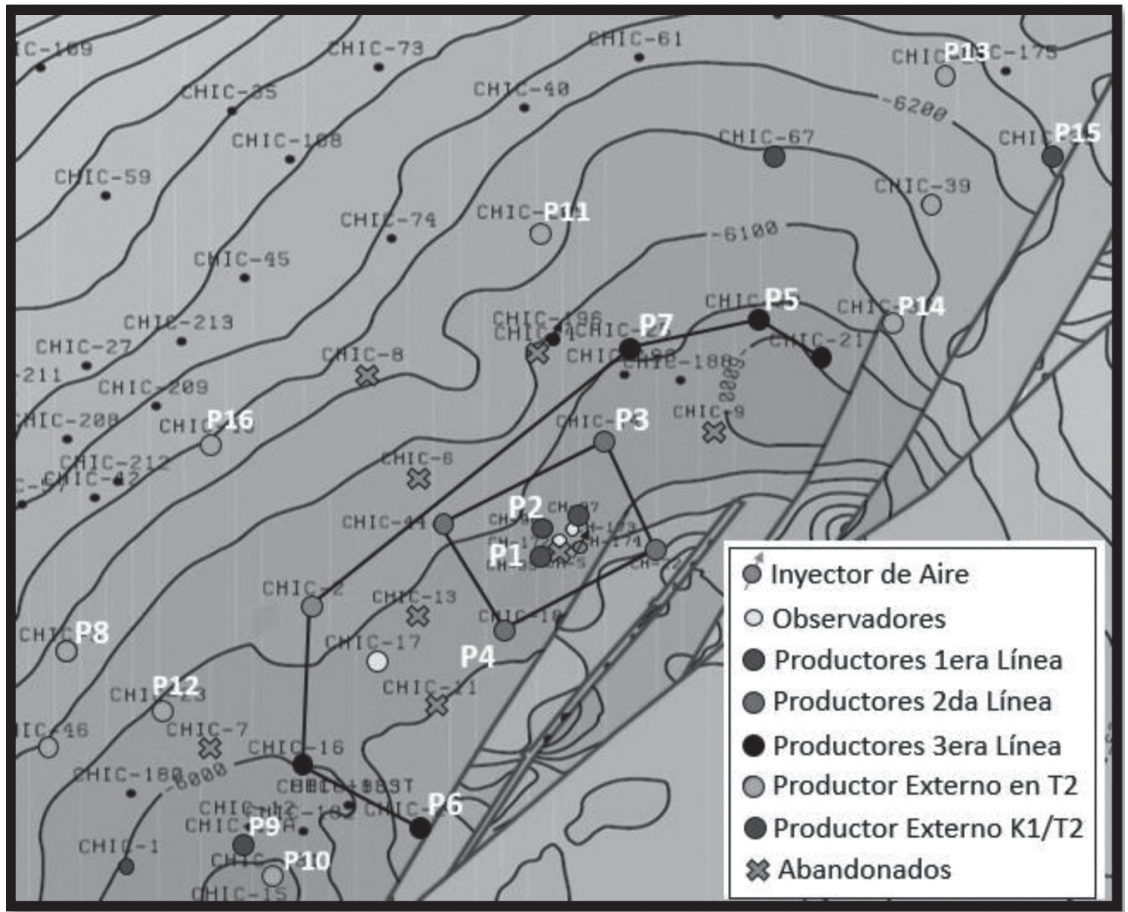

Figura 1. Arreglo de pozos del P1 al P16 en el área de influencia del PIAR.

Análisis de la composición de los gases por GC La composición de los gases se determinó usando un cromatógrafo de gases Agilent Technologies 7890A, adaptado para el análisis de gases de refinería, de acuerdo con las normas ASTM D1945, D1946 y UOP 539. El sistema cuenta con un arreglo de siete columnas y cinco válvulas, acoplado a tres detectores: un detector de ionización en llama (GC-FID) y dos detectores de conductividad térmica (GC-TCD), que permiten un análisis completo de los hidrocarburos ligeros ( $\mathrm{C} 1$ a $\mathrm{C} 6+)$ y los gases permanentes $\left(\mathrm{CO}_{2}, \mathrm{CO}, \mathrm{O}_{2}, \mathrm{~N}_{2}, \mathrm{H}_{2}\right.$ y $\mathrm{H}_{2} \mathrm{~S}$ ), empleando helio y nitrógeno como gas de arrastre. El método cromatográfico de análisis fue desarrollado en el Instituto Colombiano del Petróleo (ICP). Para su calibración, se utilizó un patrón de gas de combustión con catorce componentes y el análisis de los perfiles cromatográficos obtenidos por GC-FID y GC-TCD se realizó a través del programa OpenLab (Agilent Technologies, Inc.). Una vez obtenida la composición de los gases, se realizaron análisis estadísticos usando el software libre "Project R". Se graficó un boxplot para observar el comportamiento de los niveles de los compuestos en cada grupo de pozos y se realizó un análisis de conglomerados (AC) para evaluar las similitudes entre la composición de los gases de los pozos. Además, se hizo un análisis de componentes principales (ACP) para evaluar la variación de la composición de los gases dentro del arreglo de pozos.

\section{Resultados y discusión}

Se encontró que, para las muestras analizadas a partir del 2017, el gas producido en los pozos está constituido principalmente por metano $-\mathrm{C}_{1}$ $(68,5 \pm 7,1 \% \mathrm{Mol}), \mathrm{CO}_{2}(11,4 \pm 5,6 \% \mathrm{Mol}), \mathrm{N}_{2}(6,7 \pm 4,2$ $\% \mathrm{Mol})$ y etano $(4,3 \pm 0,7 \% \mathrm{Mol})$; en menor proporción se determinaron gases como propano, butano, pentano, hexano e hidrógeno; estos componentes son característicos de gases naturales [12]. La concentración de cada pozo se ajusta a los rangos establecidos para un gas natural según ASTM D1945-14, a excepción de $\mathrm{H}_{2} \mathrm{~S}$ y $\mathrm{O}_{2}$. componentes no detectados en los análisis realizados, como se observa en la tabla 1. La concentración de los tres gases más abundantes presentó una alta dispersión, lo cual indica la heterogeneidad de la formación. La variación de composición de estos gases se evidencia en los perfiles cromatográficos del GC-TCD de la figura 2. 
Tabla 1. Composición gas producido en pozos P3, P1 y P11.

\begin{tabular}{cllcccc}
\hline \multirow{2}{*}{ N. pico $^{\circ}$} & \multirow{2}{*}{ Detector } & Componente & \multicolumn{3}{c}{ Concentración [\%mol] } & ASTM \\
& & & P3 & P1 & P11 & D1945-14 \\
\hline 1 & TCD & $\mathrm{H}_{2}$ & 0,02 & 0,02 & 0,02 & $0,01-10$ \\
2 & TCD & $\mathrm{CO}_{2}$ & 9,24 & 8,33 & 19,06 & $0,01-20$ \\
3 & $\mathrm{TCD}$ & Metano & 76,18 & 63,47 & 63,41 & $0,01-100$ \\
4 & $\mathrm{TCD}$ & Etano & 4,17 & 4,06 & 4,11 & $0,01-100$ \\
5 & FID & Propano & 2,24 & 2,40 & 2,91 & $0,01-100$ \\
6 & FID & Isobutano & 0,66 & 0,75 & 1,12 & $0,01-10$ \\
7 & FID & n-butano & 1,13 & 1,31 & 2,01 & $0,01-10$ \\
8 & FID & Isopentano & 0,03 & 0,54 & 0,05 & $0,01-2$ \\
9 & FID & n-pentano & 0,36 & 0,47 & 0,78 & $0,01-2$ \\
10 & FID & Hexano & 0,09 & 0,06 & 0,07 & $0,01-2$ \\
11 & TCD & $\mathrm{H}_{2} S$ & - & - & - & $0,3-30$ \\
12 & TCD & $\mathrm{O}_{2}$ & - & - & - & $0,01-20$ \\
13 & TCD & $\mathrm{N}_{2}$ & 5,87 & 18,57 & 6,45 & $0,01-100$ \\
14 & TCD & CO & - & - & - & - \\
\hline
\end{tabular}
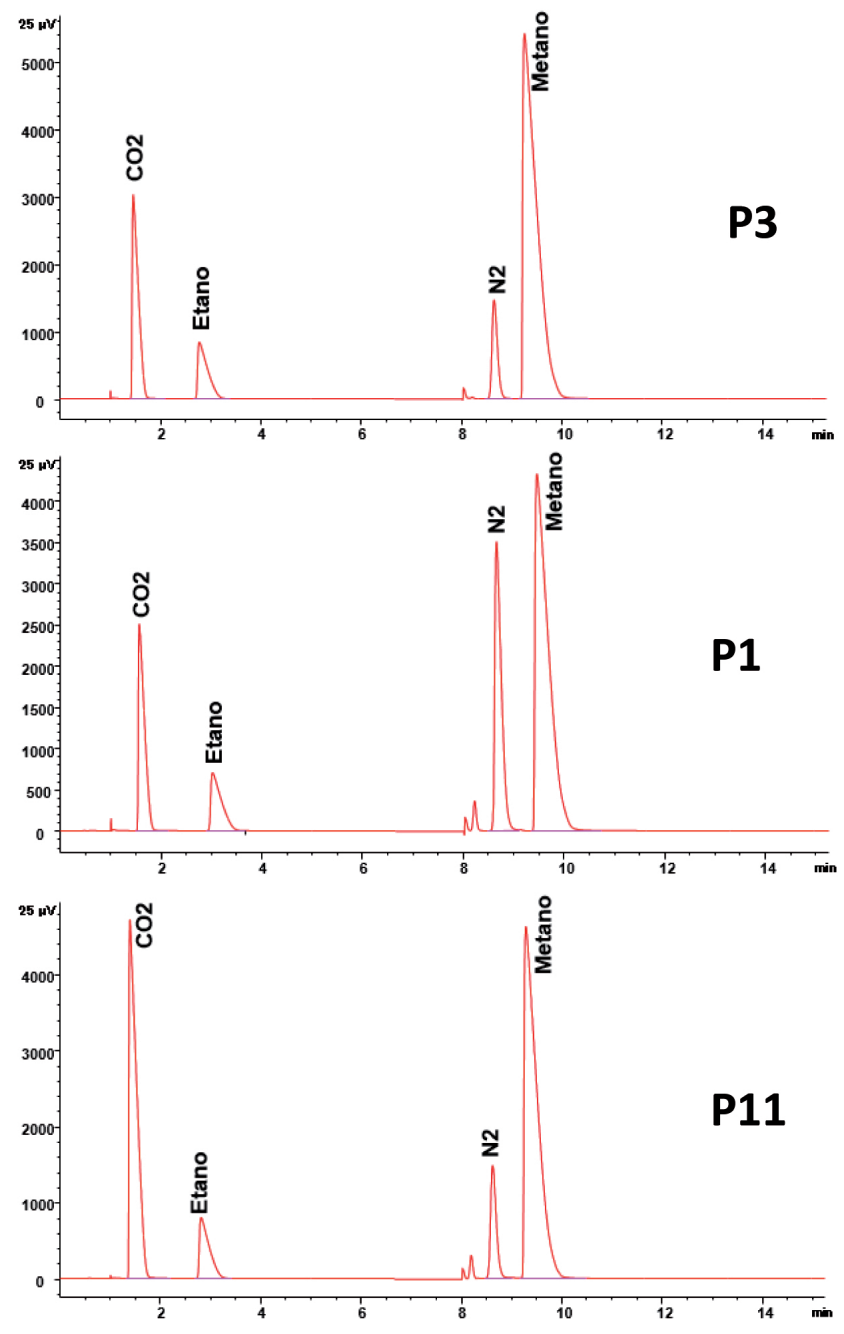

Figura 2. Perfil cromatográfíco, obtenido por GC-TCD, para los pozos P3, P1 y P11 con variación representativa en los picos correspondientes a $\mathrm{CO}_{2}, \mathrm{~N}_{2}$ y C1. 
Con el fin de evaluar la similitud de la composición de los gases de los grupos de pozos propuestos por Trujillo et al. (2018) para el PIAR, los cuales fueron organizados a partir de la NTC del 2015, se realizaron análisis boxplot por componente agrupando los pozos de primera, segunda, tercera línea y externos. Como resultado se observó una alta dispersión que indicaba que estos grupos no tenían mucha similitud, por lo que otros análisis estadísticos fueron implementados con el fin de evaluar las similitudes entre los pozos del piloto.

\section{Análisis de Componentes Principales (ACP)}

Elanálisisdecomponentesprincipalesesunatécnica estadística multivariada que se utiliza para extraer y ver información importante contenida en una matriz de datos con múltiples variables. La técnica analiza la estructura de interrelación entre un gran número de variables y resume la información en nuevas (y pocas) variables o dimensiones subyacentes comunes, conocidas como variables latentes o componentes principales, que corresponden a una combinación lineal de las variables originales, tratando de perder la mínima información posible. Hay tantos ejes o componentes principales como variables originales. La técnica se recomienda cuando las variables originales presentan alta o media correlación lineal entre ellas, es decir, ante la presencia de multicolinealidad.

El ACP es muy utilizado en estudios quimiométricos para la comparación de variables complejas, como es el caso de la composición de gas. Permite evaluar los cambios de la composición comparando los niveles por pozos y comparando cómo los componentes se afectan entre sí [14]. Como resultado, diez componentes principales (PC) fueron determinados, dentro de los cuales los dos primeros componentes PC1 y PC2 logran explicar un $62,06 \%$ de la variabilidad de la matriz de datos como se observa en la tabla 2.

Tabla 2. Resultados de la importancia de los PC generado por "Project R".

\begin{tabular}{ccccc}
\hline & PC1 & PC2 & PC3 & PC4 \\
\hline Desviación estándar & 2,0937 & 1,3500 & 1,2044 & 1,0687 \\
Proporción de varianza & 0,4384 & 0,1822 & 0,1451 & 0,1142 \\
Variación acumulada & 0,4384 & 0,6206 & 0,7657 & 0,8799 \\
\hline
\end{tabular}

Los valores de los niveles de cada compuesto fueron graficados con respecto a PC1 y PC2, como se presenta en la figura 3 . Al observarse el comportamiento de estas variables, se encuentra que altos niveles de $\mathrm{C}_{1}$ están relacionados con una disminución sobre el resto de los componentes del gas natural de la reserva. Por otro lado, los niveles de $\mathrm{N}_{2}$ y $\mathrm{CO}_{2}$ mostraron similitudes entre sí y una relación inversa con el metano $\left(C_{1}\right)$ y el etano $\left(C_{2}\right)$.

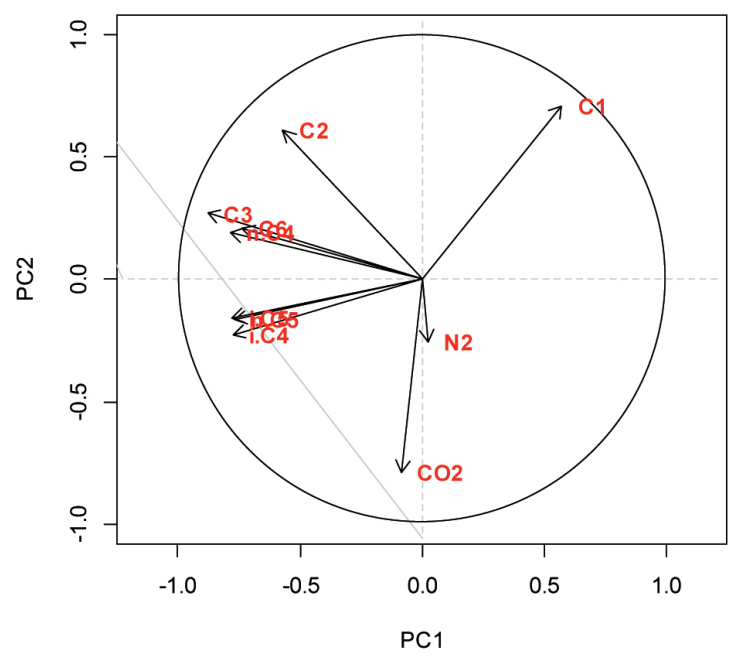

Figura 3. Gráfica ACP del comportamiento de cada variable (componente) respecto a los componentes principales CP1 y CP2 (variabilidad acumulada 0.6206). 
Por último, las fracciones de $\mathrm{HC}$ con mas de $2 \mathrm{C}$ mostraron una estrecha relación entre si, la cual es opuesta a altos niveles de $\mathrm{C}_{1}, \mathrm{~N}_{2}$ y $\mathrm{CO}_{2}$. Con base en lo anterior, se puede decir que la inyección de $\mathrm{N}_{2}$ en la PCN posiblemente causó un desplazamiento de $\mathrm{C}_{1}$ en mayor manera, lo cual puede responder al hecho de que este hidrocarburo es el mas abundante en la muestra y la dinámica de sus niveles es opuesta a la del resto de sus componentes, es decir, en pozos con mayores concentraciones de metano, se encuentran menores niveles del resto de componentes. Esta relación se hace evidente para los pozos de primera línea, pues en estos se encuentran los niveles más altos de $\mathrm{N}_{2}(\approx 12 \%)$ y al mismo tiempo los niveles más bajos de $\mathrm{C}_{1}(\approx 60 \%)$.

Del mismo modo se graficó el comportamiento de cada uno de los pozos en este mismo escenario PC1-PC2, como se observa en la figura 4. En esta se logra ver que los pozos de primera y segunda línea se encuentran muy cercanos entre sí (similares), mientras que los pozos de tercera línea y externos no muestran mucha cercanía (disímiles). A partir de esta gráfica se construyó la tabla 3 , en donde se distinguen tres posibles grupos de pozos diferenciados entre sí por presentar altas concentraciones de los compuestos más abundantes.

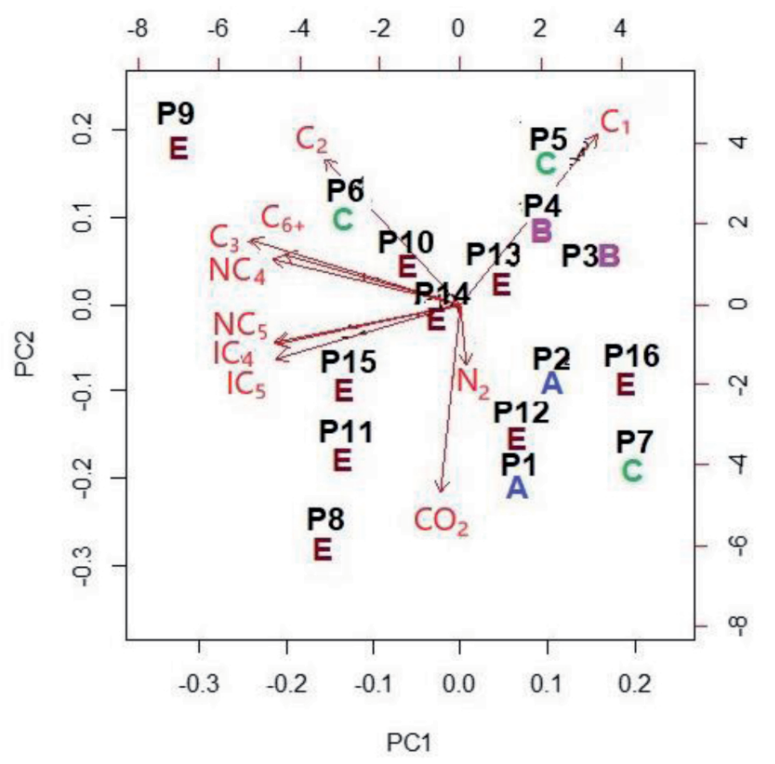

Figura 4. Gráfica ACP del comportamiento de los pozos respecto a cada una de las variables en el escenario PC1 y PC2 (varianza acumulada 0.6206). Pozos del 1 al 16. 1. ${ }^{a}$ línea (A), 2. ${ }^{a}$ línea (B), 3. ${ }^{a}$ línea (C), externos (E).

Tabla 3. Clasificación de pozos de acuerdo con su distribución en el ACP (fig. 4).

\begin{tabular}{ccc}
\hline $\begin{array}{c}\text { Componente con mayor } \\
\text { concentración }\end{array}$ & Pozos & $\begin{array}{c}\text { Ubicación según Trujillo } \\
\text { et al. (2018) [6] }\end{array}$ \\
\hline $\mathrm{C}_{1}$ & $\mathrm{P} 3, \mathrm{P} 4$ & $\begin{array}{c}\text { 2. }{ }^{\text {a línea }} \\
\text { 3. línea }\end{array}$ \\
& $\mathrm{P} 5, \mathrm{P} 6$ & Externos \\
$\mathrm{N}_{2}$ & $\mathrm{P} 9, \mathrm{P} 10, \mathrm{P} 13, \mathrm{P} 14$ & 1. a línea \\
& $\mathrm{P} 1, \mathrm{P} 2$ & 3..$^{\mathrm{a}}$ línea \\
$\mathrm{CO}_{2}$ & $\mathrm{P} 7$ & Externos \\
\hline
\end{tabular}

\section{Análisis de Conglomerados (AC)}

Complementando los resultados del ACP, se realizó un análisis clúster o de conglomerados (AC); esta técnica permite agrupar individuos u objetos con características similares. Existen clústeres jerárquicos y no jerárquicos (k-medias y k-medoids los más comunes). Los clústeres jerárquicos se crean por aglomeración (empezando con los $n$ 
objetos cada uno como un grupo, aglomerando objetos similares hasta conformar un único gran grupo) o por división (contrario al método anterior), de manera que tengan una jerarquía. El clúster jerárquico se muestra en un gráfico denominado dendograma, que permite, según la longitud de los segmentos que los unen, visualizar las diferencias entre los individuos. Para medir la similitud entre individuos u objetos se utilizan diversas medidas de similaridad. En este caso, al tener datos continuos se usó la distancia euclidea.

El AC jerárquico separó los pozos en tres grupos como se muestra en la tabla 4 , esto según sus similitudes respecto a dos componentes que explicaban un 68,4 \% de la variabilidad de los datos. El dendrograma con los grupos resultantes del $A C$ se muestra en la figura 5 . De acuerdo con los promedios de los compuestos de cada grupo, se evidencia que en el grupo 1 se hallan los mayores niveles de $\mathrm{C}_{1}$, en el grupo 2 los niveles más altos de $\mathrm{N}_{2}$ y en el grupo 3 los niveles más altos de $\mathrm{CO}_{2}$, lo cual confirma las similitudes observadas en los resultados del ACP, en la figura 4. Para los tres grupos de pozos, los porcentajes de etano $\left(\mathrm{C}_{2}\right)$ no varían significativamente, por lo que los compuestos principales son $\mathrm{C}_{1}, \mathrm{~N}_{2}$ y $\mathrm{CO}_{2}$. La nueva agrupación de los pozos según el AC se observa en la figura 6. En este mapa se logra observar que el $\mathrm{N}_{2}$ inyectado en la PCN se encuentra concentrado en los pozos cercanos al inyector, extendiéndose hacia el suroccidente del área. Por otro lado, los niveles más altos de $C_{1}$ se evidencian en los pozos que Trujillo et al. (2018) clasificaron como de segunda y tercera línea. Finalmente, hacia los pozos externos ubicados en las zonas más alejadas con respecto al pozo inyector se observan los altos niveles de $\mathrm{CO}_{2}$.

Tabla 4. Grupos de pozos dados por el AC y promedios de los niveles de los 4 componentes más abundantes para cada grupo.

\begin{tabular}{|c|c|c|c|c|c|c|c|c|c|}
\hline \multirow{3}{*}{ Grupo } & \multicolumn{5}{|c|}{ Número de Pozos } & \multicolumn{4}{|c|}{ Concentración (\%) } \\
\hline & \multicolumn{3}{|c|}{ Líneas } & \multirow{2}{*}{ Ext. } & \multirow{2}{*}{ Total } & \multirow{2}{*}{$C_{1}$} & \multirow{2}{*}{$\mathrm{CO}_{2}$} & \multirow{2}{*}{$\mathrm{N}_{2}$} & \multirow{2}{*}{$\mathrm{C}_{2}$} \\
\hline & $1 .^{a}$ & $2 .^{a}$ & 3. ${ }^{\mathrm{a}}$ & & & & & & \\
\hline 1 & 0 & 2 & 2 & 3 & 7 & 73,6 & 8,9 & 5,5 & 4,1 \\
\hline 2 & 2 & 0 & 1 & 1 & 4 & 64,6 & 7,9 & 11,6 & 4,6 \\
\hline 3 & 0 & 0 & 0 & 5 & 5 & 61,7 & 19,6 & 4,7 & 4,3 \\
\hline
\end{tabular}

Enlafigura2, dondeseobservanloscromatogramas de los pozos P1, P3 y P11, se presenta coherencia con los grupos arrojados por el AC presentados en la tabla 4. El pozo P1, perteneciente al grupo 2, presenta una concentración de $\mathrm{N}_{2}$ de 17,23 \%mol, la cual es muy alta en comparación a P3 $(5,95 \% \mathrm{~mol})$ y P11 $(6,17 \% \mathrm{~mol})$; por otro lado, el pozo P3, perteneciente al grupo 2, muestra una concentración de $\mathrm{C}_{1}$ de $77,24 \% \mathrm{~mol}$, alta en comparación a las encontradas en P1 $(58,88 \% \mathrm{~mol})$ y $\mathrm{P} 11(60,62 \% \mathrm{~mol})$. Finalmente, el P11, perteneciente al grupo 3, muestra altos niveles de $\mathrm{CO}_{2}$ con una concentración de $18,22 \%$, valor muy superior al encontrado en $\mathrm{P} 1$ $(7,73 \% \mathrm{~mol})$ y $\mathrm{P} 3(9,37 \% \mathrm{~mol})$.

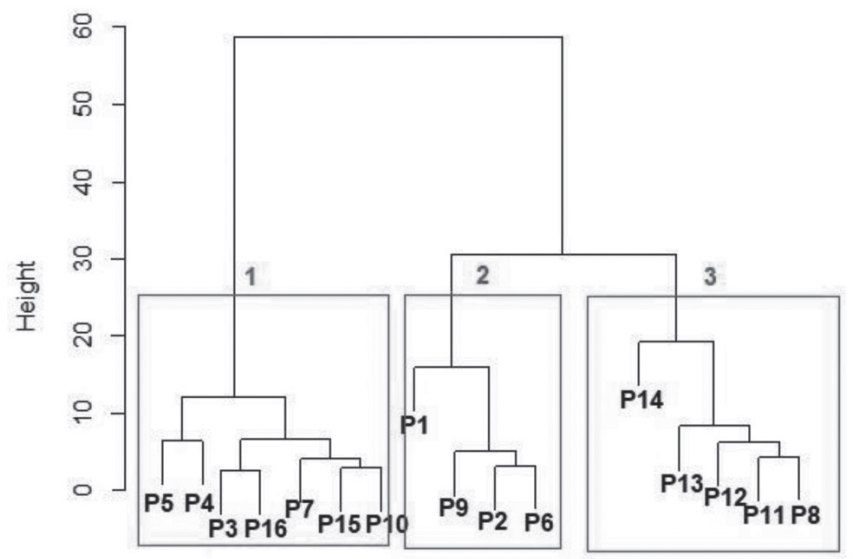

Figura 5. Dendrograma del análisis clúster. Se observan los tres grupos de pozos en cada uno de los cuadros. 


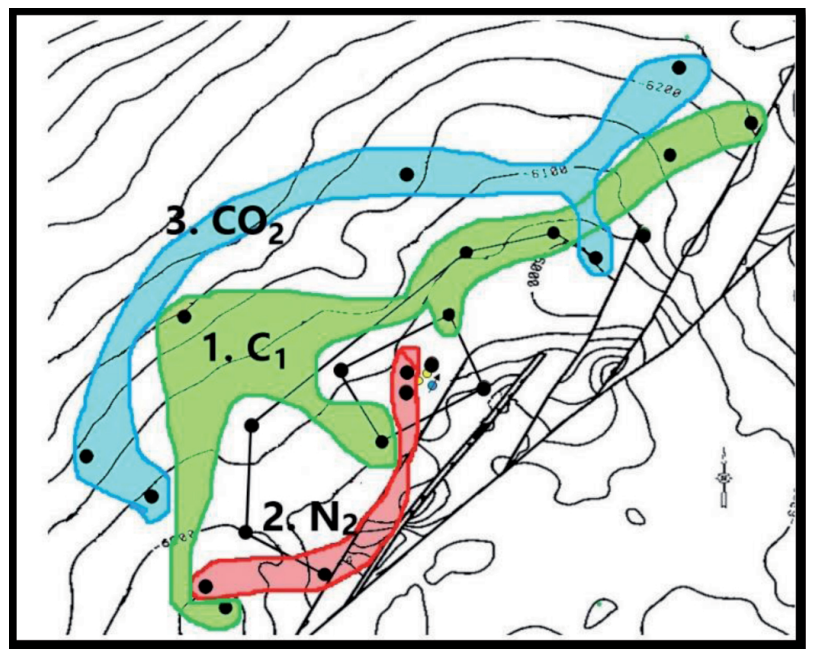

Figura 6. Zonas con mayor concentración de $\mathrm{C}_{1}, \mathrm{CO}_{2}$ y $\mathrm{N}_{2}$ en la reserva. 1. Zona con las mayores concentraciones de metano C1. 2. Zona con mayores concentraciones de Nitrógeno. 3. Zona con mayores concentraciones de $\mathrm{CO}_{2}$.

Estos nuevos grupos de pozos, según similitudes, son el punto de partida que permitirá hacer un seguimiento más preciso de la dinámica de la composición de los gases durante el desarrollo de la combustión. En vista de que esta es una variable constituida por varios compuestos individuales que se encuentran correlacionados, su seguimiento uno a uno puede no ser muy preciso. Por lo tanto, durante el proceso de combustión se debe continuar un seguimiento multivariado por medio de ACP, de manera tal que se puedan evidenciar cambios en estos grupos, analizar diferentes variables en tiempo real y tener alertas más concretas durante el proceso. Considerando la agrupación de pozos obtenida en el análisis estadístico, en la tabla 5 se presentan los datos que conforman la línea base de la composición de los gases. Durante la inyección de aire es fundamental el monitoreo principalmente de $\mathrm{N}_{2}, \mathrm{CO}_{2}, \mathrm{O}_{2}, \mathrm{H}_{2} \mathrm{~S}$ y $\mathrm{CO}$. De acuerdo con el comportamiento observado en otros pilotos de este tipo $[8,10]$, se esperan aumentos de la concentración de $\mathrm{N}_{2}$ como resultado del nitrógeno que ingresa con el aire inyectado. Esta elevación ocasionará posiblemente disminuciones en los niveles de $C_{1}$. De igual manera, sus aumentos alertarán sobre la cercanía del frente de combustión dentro del yacimiento. También se espera que los niveles de $\mathrm{CO}_{2}$ aumenten en los pozos donde se encuentra el frente de combustión, lo cual confirmará que hay ignición en la reserva.

Tabla 5. Línea base de la composición de los gases de producción de los pozos del piloto de inyección de aire. Se muestran los promedios determinados para los grupos 1, 2 y 3 resultado del análisis clúster.

\begin{tabular}{cccc}
\hline & \multicolumn{3}{c}{ Concentración (mol\%) } \\
\cline { 2 - 4 } Gas & \multicolumn{3}{c}{ Grupo } \\
\cline { 2 - 4 } & $\mathbf{1}$ & $\mathbf{2}$ & $\mathbf{3}$ \\
\hline $\mathrm{N}^{2}$ & $5,5 \pm 1,7$ & $11,9 \pm 5,9$ & $4,8 \pm 1,6$ \\
$\mathrm{CO}_{2}$ & $8,6 \pm 2,4$ & $8,3 \pm 2,3$ & $20,1 \pm 2,5$ \\
$\mathrm{C}_{1}$ & $75,8 \pm 3,9$ & $66,7 \pm 2,8$ & $63,3 \pm 5,7$ \\
$\mathrm{C}_{2}$ & $4,3 \pm 0,5$ & $4,7 \pm 1,1$ & $4,4 \pm 0,5$ \\
$\mathrm{C}_{3}$ & $2,2 \pm 0,3$ & $3,3 \pm 1,5$ & $2,9 \pm 0,7$ \\
$\mathrm{IC}_{4}$ & $0,8 \pm 0,2$ & $1,1 \pm 0,4$ & $1,2 \pm 0,7$ \\
$\mathrm{NC}_{4}$ & $1,0 \pm 0,3$ & $1,4 \pm 0,8$ & $1,3 \pm 0,5$ \\
$\mathrm{IC}_{5}$ & $0,5 \pm 0,4$ & $0,6 \pm 0,2$ & $0,6 \pm 0,3$ \\
$\mathrm{NC}_{5}$ & $0,5 \pm 0,4$ & $0,5 \pm 0,2$ & $0,5 \pm 0,3$ \\
$\mathrm{C}_{6+}$ & $0,7 \pm 0,3$ & $1,2 \pm 0,5$ & $0,7 \pm 0,4$ \\
$\mathrm{H}_{2}$ & $0,02 \pm 0,006$ & $0,02 \pm 0,005$ & $0,02 \pm 0,003$ \\
$\mathrm{Total}$ & $100 \%$ & $100 \%$ & $100 \%$ \\
\hline
\end{tabular}


El aumento significativo de $\mathrm{O}_{2}$ permitirá evaluar y regular la tasa de inyección de aire. Altas concentraciones de este gas alertarán sobre la posible formación de una atmósfera explosiva. Durante la combustión in situ a alta temperatura (HTO), los hidrocarburos reaccionan con el $\mathrm{O}_{2}$ para formar principalmente $\mathrm{CO}_{2}$, agua y energía; si esta reacción se produce a baja temperatura, los productos principales serán coque, hidrocarburos craqueados y óxidos de carbono [15]. Durante el piloto de combustión in situ ejecutado en el Campo Quifa, se observó que la composición global tenía alta concentración de $\mathrm{CO}_{2}(14,7 \%)$ mientras que la de $\mathrm{O}_{2}$ fue baja $(1,6 \%)$, indicando que las reacciones a alta temperatura (HTO) fueron dominantes durante el proceso de combustión [10]. Por otra parte, la detección de CO permitirá identificar una posible combustión incompleta dentro de la reserva, lo cual alerta sobre la tasa de inyección de aire [8]. Finalmente, la identificación de gases como $\mathrm{H}_{2} \mathrm{~S}$ permitirá establecer controles apropiados para evitar la formación de atmósferas tóxicas.

\section{Conclusiones}

Los gases analizados de los pozos de PIAR son ricos en hidrocarburos livianos, de los cuales el metano $\left(C_{1}\right)$ es el hidrocarburo en mayor concentración, seguido por $\mathrm{CO}_{2}$ y $\mathrm{N}_{2}$. El análisis estadístico mostró que tres años después de la $\mathrm{PCN}$, los gases de producción no son similares en lo grupos determinados durante esta prueba, lo que permitió reorganizar los pozos en tres grupos de acuerdo con las similitudes encontradas en la composición actual de metano, $\mathrm{N}_{2}$ y $\mathrm{CO}_{2}$. Estos nuevos grupos de pozos según similitudes son el punto de partida que permitirá hacer un seguimiento más preciso de la dinámica de la composición de los gases durante el desarrollo de la combustión.

\section{Referencias bibliográficas}

[1] Santos RG, Loh W, Bannwart AC, Trevisan OV. An overview of heavy oil properties and its recovery and transportation methods. Brazilian J. Chem. Eng. 2014;31:571-90.

[2] Peñuela Muñoz JH. Crudos pesados: la realidad del sector hidrocarburos de Colombia. Rev. VirtualPRO. 2017;(184):3

[3] Guo K, Li H, Yu Z. In-situ heavy and extra-heavy oil recovery: A review. Fuel. 2016;185:886-
902.

[4] Mahinpey N, Ambalae A, Asghari K. In situ combustion in enhanced oil recovery (EOR): a review. Chem. Eng. Commun. 2007;194:9951021.

[5] Gutierrez M, Bonilla F, Gil L, Parra W, Campo $P$, Orozco $A$, et al. Chemical stimulation pilot at a heavy-oil field: key considerations, workflow, and results. En: SPE Canada heavy oil technical conference; 2017 feb 15-16; Calgary, Alberta, Canada: Society of petroleum engineers; 2017.

[6] Trujillo M, Delgadillo C, Niz-Velásquez E, Claro $\mathrm{Y}$, Rodriguez E, Rojas R. Reducing uncertainty at the chichimene in situ combustion pilot by means of a nitrogen connectivity test. En: SPE Improved oil recovery conference; 2018 Abr 14-18; Tulsa, Oklahoma, USA: Society of petroleum engineers; 2018. p. 1-16.

[7] Dayal HS, Pandey V, Mitra S, Bhushan BV, Bhandari AC, Dwivedi MM. Monitoring of insitu combustion process in southern part of balol field through analysis of produced fluids. En: SPE Heavy oil conference and exhibition; 2010 dic 12-14; Kuwait (ciudad), Kuwait: Society of petroleum engineers; 2011.

[8] Bojes JM, Wright GB. Application of fluid analyses to the operation of an in situ combustion pilot. J. Can. Pet. Technol. 1989;28(1):106-19.

[9] Panait-Patica A, Serban D, Ilie N, Pavel L, Barsan N. Suplacu de barcau field-a case history of a successful in-situ combustion exploitation. En: SPE Europec/EAGE annual conference and exhibition; 2006 jun 12-15; Viena, Austria: Society of petroleum engineers; 2006.

[10] Gil E, Quintero NM, Rojas LA, Fuenmayor M, Farouq Ali S. Monitoring and optimization of an in-situ combustion pilot applying STAR ${ }^{\mathrm{TM}}$ technology. En: SPE Canada heavy oil technical conference; 2015 Jun 9-11; Calgary, Alberta, Canada: Society of petroleum engineers; 2015. p. 1-15.

[11] Guarin Arenas F, Garcia CA, Diaz Prada CA, Cotes Leon EDJ, Santos N. A new inflow model for extra-heavy crude oils: case study chichimene field, Colombia. En: SPE Latin American and Caribbean petroleum engineering conference; 2009 dic 1-3; Lima, Peru: Society of petroleum engineers; 2010.

[12] Faramawy S, Zaki T, Sakr AA. Journal of natural gas science and engineering natural 
gas origin, composition, and processing: a review. J. Nat. Gas Sci. Eng. 2016;34:34-54.

[13]Ferreira JEV, Pinheiro MTS, dos Santos WRS, Maia RDS. Graphical representation of chemical periodicity of main elements through boxplot. Educ. Química. 2016;27(3):209-16.

[14] University of Otago. A tutorial on Principal Components Analysis Introduction. Dunedin, Otago, Nueva Zelanda: Smith LI; 2002.

[15]Rodriguez E, Ordonez A, Comas JC, Trujillo
Portillo ML, Belgrave JDM. A framework for consolidating air injection experimental data. En: SPE Latin America and Caribbean petroleum engineering conference; $2011 \mathrm{abr}$ 16-18; Ciudad de Mexico, Mexico: Society of petroleum engineers; 2012. p. 1-9.

[16] Barrero D, Pardo A, Vargas CA, Martínez JF. Colombian sedimentary basins: Nomenclature, boundaries and petroleum geology, a new proposal. INH. 2007;1:92. 